\title{
COMPUTER SIMULATION FOR SUBCRITICAL CONVECTION IN MULTI-COMPONENT ALLOYS
}

\author{
V.V. KOLMYCHKOV, O.S. MAZHOROVA and YU.P. POPOV \\ Keldysh Institute of Applied Mathematics, Russia Academy of Sciences \\ Miusskaya Sq.4, 125047, Moscow, Russia \\ E-mail: magor@keldysh.ru
}

Received September 9, 2005; revised January 9, 2006

\begin{abstract}
Subcritical convection with hexagonal flow pattern is registered in $3 \mathrm{D}$ computer simulation of convective mass transfer in ternary solution under phase transition conditions. The calculations are evaluated by the classical theory of hydrodynamic stability and display a good agreement with linear and finite amplitude stability analysis.
\end{abstract}

Key words: convective instability, subcritical convection, computer simulation

\section{Introduction}

Numerical study of convective heat and mass transfer for crystal growth technology have developed into a distinctive and vital branch of fluid mechanics. Experimental data, as well as theoretical analysis, prove the profound influence of natural convection on the properties of grown material. Computer simulation for flow field and associated thermal and concentration distributions is widely used to optimize the configuration and operation of production facilities, to complement and interpret experimental results.

Convection usually is an undesirable factor adversely affecting the solidification conditions [22, 25]. In most cases diffusive regimes of matter transport towards the solidification front are preferable for the grown material properties. Therefore evaluation of the stability threshold for the transition from the diffusive mode to the convective one is of primary importance for the design of well controlled crystal growth techniques. Also it contributes to the stability theory of convective motion.

The paper deals with computer simulation for the onset of convection in ternary nondilute alloy under phase transition conditions. The problem arises in numerical study of liquid phase epitaxy (LPE), which is an important 
crystal growth process from both practical and fundamental viewpoints [3, $25,27]$.

In a typical setup a solution of the components $A$ and $B$ in molten $C$ is brought into contact with a substrate $A_{x} B_{1-x} C$. For definiteness, the substrate is supposed to be placed horizontally under the solution. At the initial temperature the solution is saturated and in equilibrium with the solid $A_{x} B_{1-x} C$.

The epitaxial growth is realized through the gradual system cooling. When the temperature changes, then initially saturated solution becomes supersaturated. To restore the local equilibrium at the solid/liquid interface components A, B precipitate out of the solution onto the substrate. Interface concentrations of the two phases assume values compatible with the phase diagram and mass conservation, while the bulk of the liquid retains supersaturated. The reduction in the concentration of the dissolved units in the vicinity of the growing layer gives rise to the nonlinear concentration profile and leads to density gradient normal to the substrate. It can be stable, if the solvent has a greater density than the solutes, and unstable, if the densities are differently disposed. Here we consider the latter case and suppose both components A and B contribute to instability. Concentration gradient provides a driving force for convective motion. This is the principle convective mode in LPE $[3,5,7]$.

The second mode may occur due to the gradual temperature changes. The non-dimensional parameters, characterizing the relative strength of buoyancy forces in natural convection, are solutal $\left(R a_{D_{i}}\right)$ and thermal $\left(R a_{T}\right)$ Rayleigh numbers. They are induced by concentration and temperature gradients respectively: $R a_{D_{i}}=g \beta_{i} H^{3} \Delta C_{i} /\left(D_{i} \nu\right), R a_{T}=g \beta_{T} H^{3} \Delta T /(\kappa \nu)$, where $i=\mathrm{A}, \mathrm{B}, g$ is the gravitation constant, $\beta_{i}$ is solutal expansion coefficient, $H$ is the characteristic dimension, $\nu$ is the kinematic viscosity, $D_{i}$ is the diffusion coefficient, $\Delta C_{i}$ is the characteristic concentration difference, $\kappa$ is the thermal diffusivity, $\beta_{T}$ is thermal expansion coefficient, $\Delta T$ is the characteristic temperature difference.

For reasonable operating conditions, solutal Rayleigh number varies in the range $R a_{D} \sim 10^{3} \div 10^{5}$. The thermal Rayleigh number, due to slow cooling rate and good heat conductivity of the liquid phase, is far lower than solutal one: $\frac{R a_{D_{i}}}{R a_{T}} \sim 10^{2}[3]$. It means that thermal convection is negligible. The temperature distribution is approximately uniform throughout the system, but it's value changes with time. These temperature changes do not affect the fluid flow and solute transport significantly. They displace the equilibria and alter the phase relations at the crystal-liquid interface $[5,7,13]$.

\section{Mathematical Model}

Computer simulation for LPE growth of $\mathrm{A}_{x} \mathrm{~B}_{1-\mathrm{X}} \mathrm{C}$ structures is based on the following assumptions. The growth takes place under quasi-equilibrium conditions. In particular, it means that, at the solid-liquid interface, the composition of the two phases are related by phase diagram of the system. Temperature 
throughout the system is supposed to be uniform in space and changes in time according to the prescribed rule: $T(t)=T_{0}-\alpha(t) t$, where $T_{0}$ is the initial temperature, $\alpha(t)$ is the cooling rate and $t$ is time.

The surface kinetics effects are ignored, the growth rate is not limited by the interface phenomena and controlled by bulk transport. Mass transfer in the solution is determined by diffusion and natural convection. Diffusion in the solid phase is neglected. Furthermore, as the thickness of epitaxial layer is usually within $1-2 \%$ of the solution depth, the change of the liquid volume caused by the film growth is neglected. The solution is supposed to be incompressible and the Boussinesq approximation is adopted.

Under the above assumptions, the process is described by 3D timedependent fluid flow and mass transport equations. In Cartesian coordinates $(x, y, z)$, the dimensionless governing equations take the form

$$
\begin{aligned}
& \partial_{t} V+(V \nabla) V=-\nabla p+\Delta V+\sum_{i=1}^{2} G r_{i} C_{i} e_{z}, \\
& \nabla V=0 \\
& \partial_{t} C_{i}+(V \nabla) C_{i}=\frac{1}{S c_{i}} \Delta C_{i},
\end{aligned}
$$

where

$$
\partial_{\xi} \equiv \frac{\partial}{\partial \xi}, \quad \nabla=\left(\partial_{x}, \partial_{y}, \partial_{z}\right), \quad \Delta=\nabla^{2}=\partial_{x x}^{2}+\partial_{y y}^{2}+\partial_{z z}^{2},
$$

$(x, y, z) \in \mathcal{D}, \mathcal{D}=[0, L] \times[0, L] \times[0,1], V=\left(V_{x}, V_{y}, V_{z}\right)$ is the velocity vector, $p$ is pressure, $C_{i}, i=\mathrm{A}, \mathrm{B}$ is the concentration of the correspondent component dissolved in the liquid phase, $G r_{D_{i}}=\frac{g \beta_{i} H^{3} \Delta C_{i}}{\nu^{2}}$ is the solutal Grashoff number, $S c_{i}=\nu / D_{i}$ is the Schmidt number, $e_{z}=(0,0,-1)$.

The non-dimensional variables are introduced by scaling the length with the liquid phase depth $(H)$, time is scaled with $H^{2} / \nu$ and concentration is scaled with the initial concentration of one of the components in the solution. The phase transition is going on the substrate that is placed under the solution at $z=0$. The interface conditions on concentration fields consist of the mass balance between the transported and incorporated solute species:

$$
\partial_{n} C_{i}=V_{g r} \mathrm{Sc}_{i}\left(C_{i}^{s}-C_{i}\right), \quad i=\mathrm{A}, \mathrm{B}
$$

and phase diagram representing the equilibrium between the solution and growing layer:

$$
F_{l i q}\left(C_{i}, T\right)=0, \quad F_{\text {sol }}\left(C_{i}^{s}, C_{i}, T\right)=0
$$

where $T(t)$ is temperature of the system, $C_{i}^{s}$ is concentration of the correspondent solute specie in the solid phase $V_{g r}$ - growth rate. On the remainder of the boundary: $\partial_{n} C_{i}=0$. The boundary condition for the velocity field is $V=0$. 
Table 1. Non-dimensional parameters.

\begin{tabular}{lcc}
\hline \multicolumn{1}{c}{ Parameter } & Notation & Value \\
\hline Grashoff number for component A & $G r_{\mathrm{A}}$ & $2 \div 3$ \\
Grashoff number for component B & $G r_{\mathrm{B}}$ & $17 \div 25$ \\
Schmidt number for component A & $S c_{\mathrm{A}}$ & 57 \\
Schmidt number for component B & $S c_{\mathrm{B}}$ & 57 \\
Aspect ratio & $L / H$ & $20 \div 25$ \\
\hline
\end{tabular}

Typical values of non-dimensional parameters used in our simulation are listed in the Table 1 . They correspond to $\mathrm{Cd}-\mathrm{Hg}-\mathrm{Te}$ system $[5,6,27]$. The phase diagram is described in $[4,6]$.

At $t=0$ the liquid is in the static state. Initial condition for concentration is determined by phase diagram at temperature $T_{0}$ and the substrate composition with which the liquid is in equilibrium.

\section{Model Problem}

Let us make some additional assumptions that clear up a link between the process under consideration and known problems concerning the onset of finite amplitude convection in horizontal layer with nonlinear undisturbed profile of active scalar quantity.

We neglect the existence of component A with the smaller Grashoff number in the body force term, and suppose that it does not influence the solubility of the component $\mathrm{B}$ in the melted component $\mathrm{C}$. Also we assume the linearity of the phase diagram and write it in the following form:

$$
C_{\mathrm{B}}=C_{\mathrm{B}}^{0}+\lambda(T)\left(T-T_{0}\right), \quad \lambda(T)>0,
$$

where $\lambda(T)$ is the slope of phase diagram. While the solution temperature changes in time at a given rate $\alpha$, the equilibrium concentration at the interface is represented by

$$
\left.C_{\mathrm{B}}\right|_{z=0}=C_{\mathrm{B}}^{0}+\tilde{\eta} t, \quad \tilde{\eta}=-\alpha \lambda(T) .
$$

All these assumptions reduce (2.1)-(2.5) to the standard problem of natural convection with respect to concentration $C_{\mathrm{B}}$ as the active scalar quantity:

$$
\left\{\begin{array}{l}
\partial_{t} V+(V \nabla) V=-\nabla p+\Delta V+G r Q e_{z}, \\
\nabla V=0 \\
\partial_{t} Q+(V \nabla) Q=\frac{1}{P} \Delta Q
\end{array}\right.
$$

where $Q$ is the active scalar, $G r=G r_{\mathrm{B}}, P$ is the Schmidt number (for thermal convection it would be the Prandtl number). The boundary condition for $Q$ at $z=0$ is 


$$
\left.Q\right|_{z=0}=Q^{(0)}+\tilde{\eta} t
$$

on the remainder of the boundary $\frac{\partial Q}{\partial \mathrm{n}}=0$.

In the static case the solution of (3.1)-(3.2) establishes at

$$
\widetilde{Q}(t, z)=Q^{(0)}+\tilde{\eta} t+\frac{\tilde{\eta} P}{2} z(z-2) .
$$

Our goal is to study convective instability in the horizontal fluid layer with nonlinear undisturbed profile of active scalar (3.3).

The deep insight into the problem is given in $[17,18]$. R.Krishnamurty performed the finite amplitude stability analysis for an infinite horizontal layer of fluid $(0<z<1)$ with the mean temperature changing steadily at a given rate. Upper and lower surfaces were rigid and perfectly conducting. The boundary condition for the Boussinesq equations were the following:

$$
\begin{gathered}
V=0, \quad z=0, \quad z=1, \\
\left.Q\right|_{z=0}=+\frac{1}{2} \delta Q+\eta t, \quad z=0, \\
\left.Q\right|_{z=1}=-\frac{1}{2} \delta Q+\eta t, \quad z=1 .
\end{gathered}
$$

The static state solution that satisfies (3.5) is given by:

$$
\widetilde{Q}(t, z)=Q^{(0)}+\eta t-\delta Q\left(z-\frac{1}{2}\right)+\frac{\eta P}{2} z(z-1),
$$

where $\delta Q=\left.Q\right|_{z=0}-\left.Q\right|_{z=1}$. This example represents the case in which the conduction temperature profile is parabolic, its shape does not depend on time, while all points of the liquid are changing with respect to temperature at the same rate $\eta$ as the boundary values. The sign and the absolute magnitude of $\eta$ define the direction of the profile curvature and the curvature itself, respectively.

Profile (3.6) is similar to (3.3). In epitaxial growth, the concentration plays the role of active scalar quantity instead of temperature. Its evolution at the interface is governed by the parameter $\tilde{\eta}=\lambda(T) \alpha, \tilde{\eta}$ can be treated as the temperature changing rate $\eta$ in [17]. The analogy allows us to use the finite amplitude analysis and experimental results from $[17,18]$ to assess our numerical results. For the sake of self-containment, a brief overview of the stability studies concerning the problem under consideration is given below. The details can be found in [17].

\section{On the Finite Amplitude Stability}

Analysis of finite amplitude convection is based on the expansion of stationary solution of the governing equations (3.1) and the Rayleigh number in series of powers of amplitude parameter $\varepsilon$ and parameter $\eta$, that are responsible for the 
nonlinearity of undisturbed active scalar profile. Both parameters supposed to be finite but small. In our case the active scalar can be written as

$$
Q(t, x, y, z)=\widetilde{Q}(t, z)+\theta(x, y, z) .
$$

The stationary fields and the Rayleigh number then read:

$$
\begin{aligned}
& V(x, y, z)=\sum_{n=1, m=0}^{\infty} \varepsilon^{n} \eta^{m} V^{(n, m)}(x, y, z), \\
& \theta(x, y, z)=\sum_{n=1, m=0}^{\infty} \varepsilon^{n} \eta^{m} \theta^{(n, m)}(x, y, z), \\
& R a=\sum_{n=0, m=0}^{\infty} \varepsilon^{n} \eta^{m} R^{(n, m)} .
\end{aligned}
$$

The expansions (4.1)-(4.3) are substituted into the governing equations and using $\varepsilon$ and $\eta$ as ordering parameters an infinite set of linear inhomogeneous equations is obtained. The problem of ordering the non-linearities by the expansion in powers of $\varepsilon$ and the convergence of the expansion were studied in [20,23]. The coefficients $R^{(n, m)}$ are determined in such a way as to satisfy the solubility conditions of the inhomogeneous equations. Thus $\eta$ appears as a given small parameter in equations having known solutions when $\eta=0$.

The lowest order terms in (4.1)-(4.2) $V^{(1,0)}, \theta^{(1,0)}$ are solutions of linear stability problem for the linear undisturbed profile. The vertical component of the velocity is given in the following form

$$
V_{z}^{(1,0)}=f(z) w(x, y), \quad w(x, y)=\sum_{n=-N}^{N} A_{n} \exp \left(i \mathbf{k}_{\mathbf{n}} \cdot \mathbf{r}\right)
$$

Here $\mathbf{r}$ is a horizontal position vector, $\mathbf{k}_{\mathbf{n}}$ is a horizontal wave vector, $w(x, y)$ represents the plan form of the stationary critical motion $[9,10] . R^{(0,0)}$ in $(4.3)$ is the well known critical Rayleigh number equal to the minimum eigenvalue of the linear stability problem. It determines the point at which the static layer becomes unstable. For the case of two rigid boundaries $R^{(0,0)}=R_{c}=1707.8$ $[9,10]$.

Calculations of the succeeding series terms performed in [17] give $R^{(1,0)}=$ $R^{(0,1)}=0$ and produce the following expression for the Rayleigh number:

$$
R a=R^{(0,0)}+\eta^{2} R^{(0,2)}+\varepsilon \eta R^{(1,1)}+\varepsilon^{2} R^{(2,0)}+\ldots,
$$

where $R^{(0,2)}<0, R^{(2,0)}>0$. For hexagonal planform $R^{(1,1)}>0$, and $R^{(1,1)}=0$ for all the other plan forms.

The main consequences from the representation (4.5) are the following.

- The critical Rayleigh number corresponding to a nonlinear undisturbed profile $(\eta \neq 0)$ is smaller than for the linear profile $(\eta=0$.) In the limit case taking an infinitesimal amplitude we get 


$$
R_{c}=R^{(0,0)}+\eta^{2} R^{(0,2)}, \quad R^{(0,2)}<0 .
$$

For example, in the case of two rigid boundaries $R_{c}=1537.5$ for nondimensional parameter $\eta=-8$ (see [17]).

- For all plan forms, except for hexagons, linear stability theory should correctly predict the lowest Rayleigh number at which the fluid starts to be unstable. Taking into account that $R^{(1,1)}=0\left(R^{(1,1)} \neq 0\right.$ only for hexagonal plan form), it can be found that $R a-R_{c}=\varepsilon^{2} R^{(2,0)}$. Since $R^{(2,0)}$ is positive, a real amplitude $\varepsilon$ is impossible at

$$
R a<R_{c}, \quad|\varepsilon| \sim \sqrt{R a-R_{c}}
$$

and liquid is stable at $R a<R_{c}$ (see Fig. 1).

- For hexagonal plan form $\left(R^{(1,1)} \neq 0\right)$, the motion is possible below $R_{c}$. The static state is unstable with respect to finite amplitude disturbances, since infinitesimal perturbations are known to decay below $R_{c}$. Solving (4.5) for $\varepsilon$ gives the following solution:

$$
\varepsilon=-\frac{\eta R^{(1,1)}}{2 R^{(2,0)}} \pm \sqrt{\left(\frac{\eta R^{(1,1)}}{2 R^{(2,0)}}\right)^{2}+\frac{R a-R_{c}}{R^{(2,0)}}} .
$$

The minimum Rayleigh number for which $\varepsilon$ exists is given by

$$
R_{\text {min }}=R_{c}-\frac{\left(\eta R^{(1,1)}\right)^{2}}{4 R^{(2,0)}},
$$

and the corresponding value of the amplitude parameter is equal to

$$
\varepsilon_{\min }=-\frac{\eta R^{(1,1)}}{2 R^{(2,0)}} .
$$

- As it is clear from (4.9) the direction of the flow in hexagonal cell is determined by the sign of $\eta$. If $\eta<0$, then the direction of the flow in the center of hexagonal cell is upward, i.e. the cells are of $l$-type, and it is downward if $\eta>0$, i.e. the cells are of $g$-type.

In order to be obtained in experiments, the steady state finite amplitude solution must be stable at least with respect to disturbances of infinitesimal amplitude. The stability of the finite amplitude flows with parabolic undisturbed profile was investigated in [17]. Qualitatively the results are the same as F.H.Busse has obtained studying finite amplitude convection in the fluid with varying physical properties [2]. The results are summarized in fig. 1.

If the Rayleigh number increases, the convection starts growing at the critical Rayleigh number $R_{C}$ and at finite amplitude value we get a flow in the form of stable stationary hexagon. The direction of the motion is determined by the sign of $\eta$. In the case of linear undisturbed profile, two-dimensional rolls are known to be the unique stable solution near the critical Rayleigh number [23]. For nonlinear profiles rolls are unstable in the range $\mathrm{R}_{\mathrm{C}}<R a<\mathrm{R}_{\mathrm{h}}$. At higher Rayleigh number $R a>R_{r}$ hexagons become unstable and rolls 


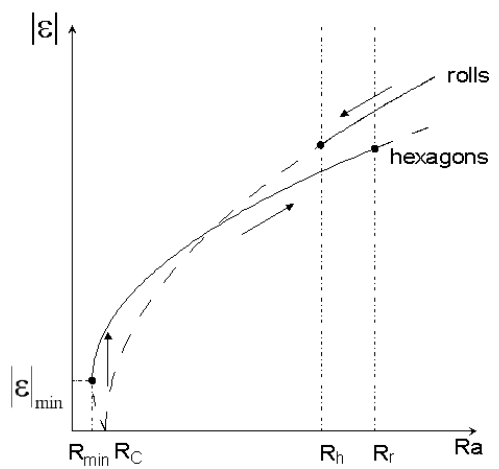

Figure 1. Qualitative dependence of the amplitude on the Rayleigh number for rolls and hexagons. Solid lines correspond to stable states, dashed ones to unstable states [2].

are found to be the stable flow. If the Rayleigh number is decreased, then a transition from rolls to hexagons occurs at $R_{h}$ and convection decays when $R a=\mathrm{R}_{\min }$ is passed. Thus there is the hysteresis effect, because the convection at a certain Rayleigh number depends on the way in which the Rayleigh number has been reached [2].

The experimental test with the fluid in the rectangular parallelepiped with aspect ratio $15 \times 15 \times 1$ confirms the theoretical results [18]. When the mean temperature was held near constant value (undisturbed profile is approximately linear), two-dimensional rolls were found to be stable flow pattern near the critical Rayleigh number. The subcritical convection in the form of hexagonal cells was observed while the mean temperature changed at a constant rate $\eta$. In particular, for non-dimensional value $|\eta| \sim 5 \div 10$, a change up to $40 \%$ from the critical number has been established. The direction of flow at the center of the hexagon was downward for positive $\eta$ and upward if $\eta$ was negative.

Though in real crystal growth process the value of the active scalar function changes at the boundary with a varying rate $\tilde{\eta}$ and there is no stationary solution, it is interesting to obtain the Rayleigh number for the onset of convective motion, i.e. the Rayleigh number beyond which the steady state would become unstable, and to compare it with theoretical predictions. The result would also have a significant practical value, because diffusion limited mass transfer in liquid phase epitaxy is favorable for the material quality $[5,6]$. For this purpose within the scope of the model described in section 2, direct numerical simulation of the process has been carried out.

\section{Numerical Procedure}

We apply a sequential procedure to solve the governing equations (2.1)-(2.3). A new value of the velocity is computed using the species concentration from 
the previous time layer in the force term. The calculated velocity field is inserted into the mass transfer equations that are solved with respect to concentrations $\left(C_{\mathrm{A}}, C_{\mathrm{B}}\right)$. The problem is approximated at staggered grid [8] using control-volume approach. Discretization is conservative for kinetic energy and concentration of dissolved species. The finite-volume scheme is implicit and it has second order accuracy in space and first order in time [12].

The Navier-Stokes equations (2.1)-(2.2) are solved by the projection method with pressure correction $[1,14]$.

Preliminary step. A preliminary pressure $\widetilde{p}$ is computed from the Poisson equation with Neumann boundary conditions:

$$
\left\{\begin{array}{l}
\Delta \widetilde{p}=\operatorname{div} F^{n} \text { in } D_{h}, \\
\frac{\partial \widetilde{p}}{\partial n}=0 \text { on } \partial D_{h}
\end{array}\right.
$$

here $F^{n}$ is the gravity term computed by using the concentration from the previous $n$-th time layer. This step corrects the temporal evolution of the pressure in accordance with the given concentration field.

Prediction step. A predicted velocity field $\widetilde{V}$ is computed implicitly from equation (2.1) in which the pressure gradient at the current time step $t_{n+1}$ is replaced by the preliminary pressure:

$$
\left\{\begin{array}{l}
\frac{\widetilde{V}-V^{n}}{\tau}+\left(V^{n} \nabla\right) \widetilde{V}=-\nabla \widetilde{p}+\Delta \widetilde{V}+F^{n} \text { in } D_{h}, \\
\widetilde{V}=0 \text { on } \partial D_{h} .
\end{array}\right.
$$

The set of algebraic equations for each component of the momentum is solved in turn, treating the grid point values of the dominant velocity component as a sole set of unknowns. To determine the velocity we use the conjugate gradient method [26].

Projection step. The velocity field $\widetilde{V}$ is corrected by taking into account the pressure gradient at time $t_{n+1}$ in order to satisfy the incompressibility constraint:

$$
V^{n+1}=\widetilde{V}-\tau \nabla\left(p^{n+1}-\widetilde{p}\right) .
$$

This step is performed by computing the pressure correction $\delta p=p^{n+1}-\tilde{p}$ from the Poisson boundary value problem:

$$
\left\{\begin{array}{l}
\Delta(\delta p)=\frac{\operatorname{div} \tilde{V}}{\tau} \text { in } D_{h}, \\
\frac{\partial \delta p}{\partial n}=0 . \text { on } \partial D_{h} .
\end{array}\right.
$$

The final velocity and pressure fields then are defined as: 


$$
\begin{aligned}
& \left\{\begin{array}{l}
V^{n+1}=\widetilde{V}-\tau \nabla \delta p \text { in } D_{h}, \\
V^{n+1}=0 \text { on } \partial D_{h},
\end{array}\right. \\
& p^{n+1}=\widetilde{p}+\delta p \text { in } D_{h} .
\end{aligned}
$$

The calculated velocity field is inserted into the mass transfer equations. Being independent inside the region, they are coupled at the solid-liquid interface by mass balance conditions (2.4) and phase diagram (2.5). Attempts to decouple them into some sequential procedure or by using iterations produced highly unreliable procedures with poor if any convergence [21]. To overcome these difficulties, we use the algorithm based on the coupled solution of mass transfer equations with respect to the concentration of both species and the Newton method is used to deal with nonlinearity in boundary conditions. The linear systems obtained at each Newton iteration are solved by the conjugate gradient method. This approach has been used successfully in $2 \mathrm{D}$ case $[5,6]$ and here we have extended it to $3 \mathrm{D}$ problems.

\section{Numerical Results}

The onset of convection is studied in the domain where the length of horizontal sides is $L=25 \mathrm{~mm}$, the vertical size changes in the range $H=0.5 \mathrm{~mm} \div 1.2$ $\mathrm{mm}$. The aspect ratio of the domain is more than 20 . In such a case the critical Rayleigh number determined for the infinite layer should be close to one for the finite region [24]. To analyze the results we introduce the viscosity and diffusion time scales as $t_{\nu}=H^{2} / \nu$ and $t_{D_{\mathrm{B}}}=H^{2} / D_{\mathrm{B}}$ respectively. The actual Rayleigh number is time dependent and it is based on the averaged concentration difference observed in the calculations $R a(t)=\frac{g \beta_{\mathrm{B}} H^{3} \delta C_{\mathrm{B}}}{D_{\mathrm{B}} \nu}$. The average difference for component $B$ is determined as

$$
\delta C_{\mathrm{B}}=\left\langle C_{\mathrm{B}}(H, t)\right\rangle-\left\langle C_{\mathrm{B}}(0, t)\right\rangle, \quad\left\langle C_{\mathrm{B}}(z, t)\right\rangle=\left\|C_{\mathrm{B}}(z, t)\right\|_{\mathcal{L}_{1}^{h}(x, y)} / L^{2} .
$$

In these runs we keep the cooling rate constant $\alpha=0.25 \mathrm{~K} / \mathrm{min}$, and change the liquid depth.

Initially the fluid is at rest, its composition is uniform through out the space and in equilibrium with the substrate. The transition to unsteadiness is allowed to develop from the actual noise that is presented in calculations. A grid of size $50 \times 50 \times 15$ and time step $\tau=0.5 t_{\nu}$ are usually used. Such mesh provide a good compromise between the CPU time and calculation accuracy.

To begin with it is worth to establish a critical depth for the onset of convection in $2 \mathrm{D}$ case. Under the same operating conditions (substrate length, cooling rate, phase diagram), convection was determined at $H=1.2 \mathrm{~mm}$ in the form of steady rolls $[4,5]$. The subcritical convection is essentially $3 \mathrm{D}$ process and it is impossible to get it in $2 \mathrm{D}$ approach, only rolls are admissible. Thus a linear stability theory with infinitesimal amplitude is valid and the lowest Rayleigh number beyond which the flow becomes unsteady is predicted 
by (4.6). In our calculation we have obtained $\eta \approx-4 \div-5 R a_{c}=R a_{c}^{2 D}=$ 1545. This value is unexpectedly close to $R a_{c}=1537.5$ computed from the truncated expression (4.6) in [17]. Though the latter value was obtained for steady solution at $\eta=-8$.

In $3 \mathrm{D}$ case, calculations show the static state for $H \leq 1 \mathrm{~mm}$. We observed the onset of convection at $H=1.1 \mathrm{~mm}$. The temporal evolution of the kinetic energy and actual Rayleigh number are plotted in Fig. 2.

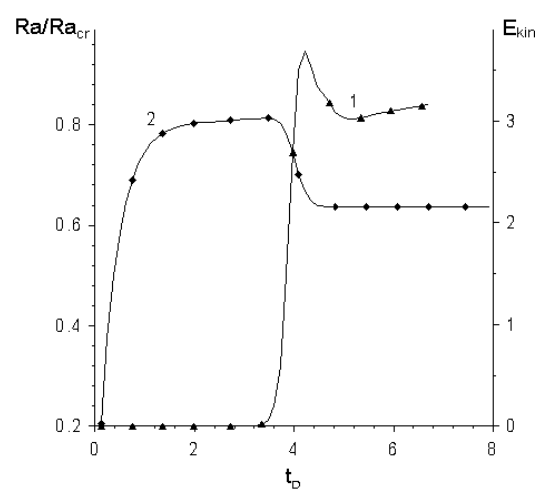

Figure 2. Time evolution of kinetic energy $E_{k i n}(t)$ (line 1$)$ and $R a(t) / R a_{c r}, R a_{c r}=$ $R a_{c}^{2 D}=1545$ (line 2).

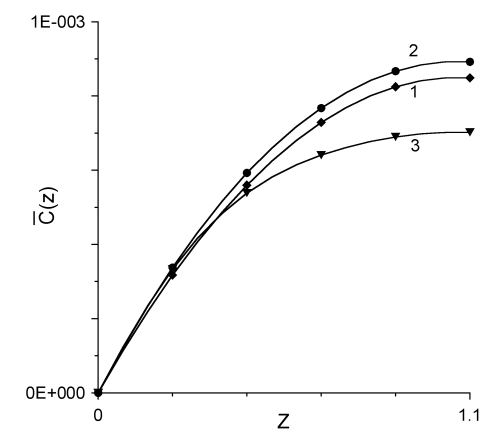

Figure 3. Distribution of $\bar{C}(z)=\left\langle C_{\mathrm{B}}(z, t)\right\rangle-\left\langle C_{\mathrm{B}}(0, t)\right\rangle$ versus $z$ for time moments $t=1.5 t_{D}$ (line 1), for $t=4.5 t_{D}$ (line 2), for $t=7.5 t_{D}$ (line 3 ).

An unstructured pattern of weak flow was firstly registered at $t=2.1 t_{D}$. At the initial stage of the process: diffusive mass transfer and initiation of convection, (Fig. 3, lines 1,2) concentration difference $\delta C_{\mathrm{B}}$ increases with time. Actual Rayleigh number also increases and reaches the value 1260. After the initiation, between time moments $2.1 t_{D}$ and $3 t_{D}$, convection slowly grows up. There is a short period of stability for the Rayleigh number which is followed 
by its decline. This is an eventual response to quick increase in kinetic energy at $3 t_{D}<t<4.2 t_{D}$ : developing convection produces a better solute stirring, hence $\delta C_{\mathrm{B}}$ (Fig. 3, line 3) and $R a$ (Fig. 2) decrease. The Rayleigh number drops down to the value approximately 1000 . It is about $40 \%$ below the well known critical point $\mathrm{Ra}=1708$ (it corresponds to $\eta=0$,) and $35 \%$ less than the value predicted by liner analysis [18] and 2D calculation [5]. Convection continues in a finite-amplitude subcritical state with asymptotically constant Rayleigh number.

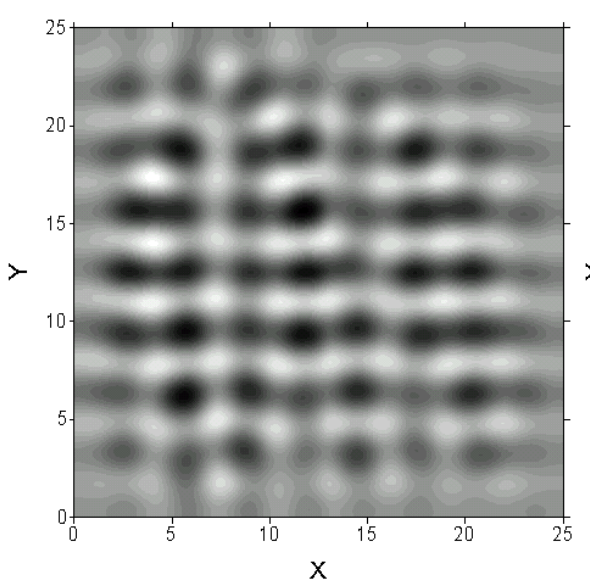

a) time $=4.5 t_{D}$

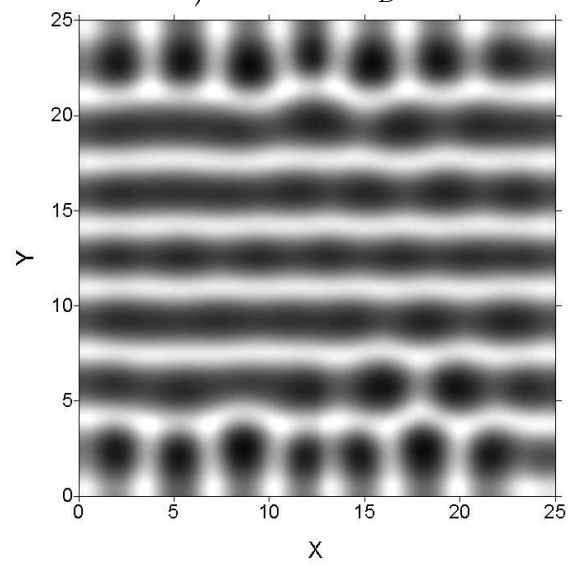

c) time $=12.3 t_{D}$

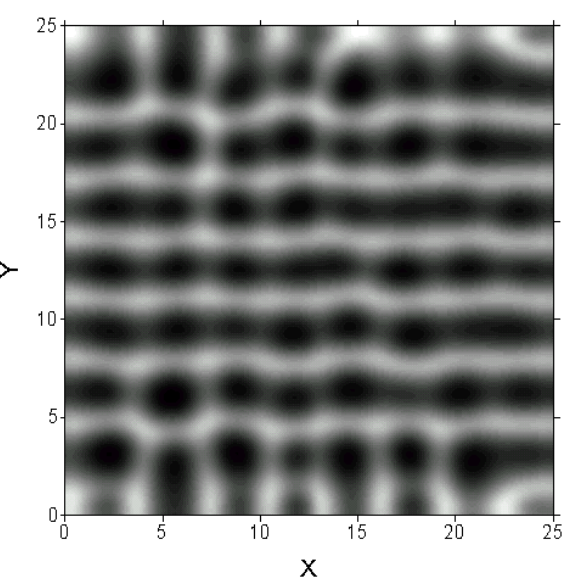

b) time $=6 t_{D}$

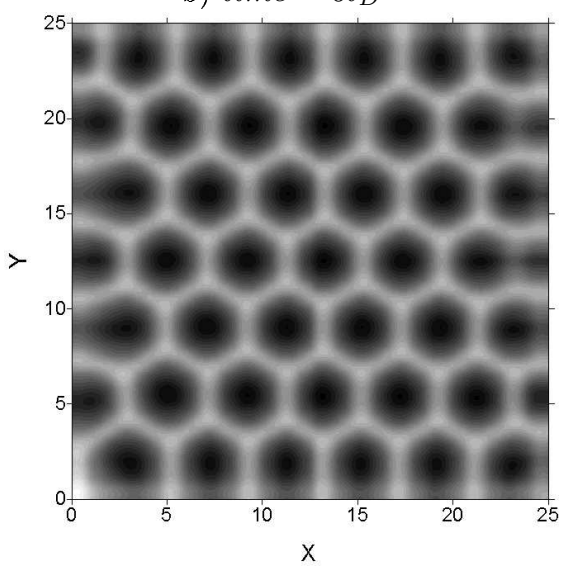

d) time $=37 t_{D}$

Figure 4. Flow patterns. Concentration field, view from the top.

Fig. 4 illustrates the evolution of the flow structure. Near $t=4.2 t_{D}$, when the kinetic energy reaches its maximum value, the flow deposits in a definite pattern. It consists of a set of primary rolls with a weaker perpendicular set of secondary ones (a bimodal pattern [28]). The intersection of the rolls produces 
structure similar to square cells with fluid rising in the center and falling at the peripheries of the cell. (Fig. 4a)

After the kinetic energy passes its maximum value, a transition from bimodal convection to rolls begins. The down flow in some sides of the quadrilaterals weakens forming fragments of slightly curved rolls. Fig. $4 \mathrm{~b}$ shows such intermediate pattern. There is a general rule, that rolls should approach a vertical boundary at a right angle [11, 19, 29]. So at the corners of the domain one can see segmented rolls, that are curved to adjoin both sides of the domain at a right angle.

The fully-developed flow in the form of rolls establishes near $t=12.3 t_{D}$ (see Fig. 4c). At this time practically the whole layer is covered with a roll pattern. Convection remains as cells confine to the two edges of the domain. Then the transition from rolls to hexagons begins. The evolution of the plan form is very slow. The final pattern of uniform hexagonal cells is established approximately at $t=37 t_{D}$ (see Fig. $4 \mathrm{~d}$ ). Distortions of the structure are observed only at the edges where imperfect packing occurs. In the dark spots the concentration of dissolved species is smaller, the solution is lighter and moves upward. Bright sides of hexagons correspond to heavier liquid where concentration is larger and the flow is downward (the cells of $l$-type). The flow pattern is stable and observed up to $t=96 t_{D}$.

Thus, according to linear stability theory, in full scale computer simulation of LPE growth, after initiation the convective motion shows the tendency to two-dimensional rolls. The growing amplitude of the motion and increasing curvature of undisturbed concentration profile makes rolls unstable and they broke down into hexagon, that is the only stable flow pattern near the critical Rayleigh number. The direction of the circulation in the convective cell coincides with theoretical predictions [17].

The results of our computations can be interpreted as finite amplitude instability occurring at Rayleigh number below that predicted by linear theory. The onset of hexagon flow pattern in our simulation is a long term process, that is also essential to subcritical convection [28].

Initiation of convection in the form of rolls with ongoing transition to hexagons attributes both to hysteresis gap and evolution of concentration profile, because we approach the critical point with decreasing Rayleigh number (see Fig. 2), while solidification increases the profile curvature. The last means that $\eta$ becomes larger and convection in hexagonal form appears to be preferred $[2,17,18]$.

In order to validate our numerical results, a series of calculations on the refined grids has been done. The spatial resolution has been doubled, while the time step has been decreased two and four times. Computations have shown different time thresholds for convection when the grid size is varied. Such result should be expected since convection develops from the noise presented in calculations. At the same time comparison of the flow patterns and integral properties of the solution, such as kinetic energy of the motion, time history of Rayleigh number are not affected by grid refinement $[15,16]$. 


\section{Conclusions}

Finite amplitude subcritical convection has been registered in a full scale 3D computer simulation for liquid phase epitaxy of ternary compounds. The calculations are compared with theoretical results on the stability of horizontal layer of fluid with nonlinear undisturbed profile of active scalar quantity. The convection starts in the form of rolls at Rayleigh number near the critical one. In our simulation, roll flow pattern, as it should be according to finite amplitude stability analysis, appears to be unstable and breaks down into stable hexagonal flow. The hexagons have been registered at Rayleigh number less than predicted by linear theory. The direction of flow circulation in the cell as well as the lowest Rayleigh number at which the static state becomes unstable also correspond to the finite amplitude stability analysis.

\section{References}

[1] O.M. Belotserkovskii. Numerical modelling in mechanics of continua. Nauka, Moscow, 1984. (in Russian)

[2] F.H. Busse. The stability of finite amplitude cellular convection and its relation to an extremum principle. Journal of Fluid Mechanics, 30, 625 - 649, 1967.

[3] I. Crossley and M.B. Small. The physical processes occurring during liquid epitaxial growth. Journal of Crystal Growth, 27(1), 35 - 48, 1974.

[4] I.A. Denisov, V.M. Lakeenkov, O.S. Mazhorova and Yu.P. Popov. Mathematical modelling for liquid phase epitaxy of $\mathrm{Cd}_{\mathrm{x}} \mathrm{Hg}_{1-x} \mathrm{Te}$ solid solution. The Keldysh Institute of Applied Mathematics, Preprint 65, Moscow, 1992. (in Russian)

[5] I.A. Denisov, V.M. Lakeenkov, O.S. Mazhorova and Yu.P. Popov. Numerical study for liquid phase epitaxy of $\mathrm{Cd}_{\mathrm{x}} \mathrm{Hg}_{1-\mathrm{x}} \mathrm{Te}$ solid solution. Journal of Crystal Growth, 245(1), 21 - 30, 2002.

[6] I.A. Denisov, O.S. Mazhorova, Yu.P. Popov and N.A.Smirnova. Numerical modelling for convection in growth/dissolution of solid solution $\mathrm{Cd}_{\mathrm{x}} \mathrm{Hg}_{1-\mathrm{x}} \mathrm{Te}$ by liquid phase epitaxy. Journal of Crystal Growth, 269(2), 284 - 291, 2004.

[7] L.A. Dmitrieva, O.S. Mazhorova, Yu.P. Popov, E.A. Tvirova and A.A. Shlenskii. Mathematical Modelling. Production of Bulk Crystals and Semiconductor Structures. Computer simulation for solutal convection in liquid phase epitaxial growth of semiconductor materials, ed. A.A. Samarski, Yu.P. Popov, O.S.Mazhorova. Nauka, Moscow, 1986. (in Russian)

[8] K. Fletcher. Computational techniques for fluid dynamics, volume 1,2. Mir, Moscow, 1991. (in Russian)

[9] G.Z. Gershuni and E.M. Zhukhovitskii. Convective stability of incompressible fluids. Nauka, Moscow, 1972. (in Russian)

[10] A.V. Getling. Rayleigh-Benard convection: structures and dynamics. World Scientific, Singapore-London, 1998.

[11] J.P. Gollub, A.R. McCarriar and J.F. Steinman. Convective pattern evolution and secondary instabilities. Journal of Fluid Mechanics, 125, 259 - 281, 1982.

[12] A.L. Goncharov and I.V. Friazinov. Finite difference schemes on nine-points stencil for Navier-Stokes equations velocity-pressure variables. The Keldysh Institute of Applied Mathematics, Preprint 53, Moscow, 1987. (in Russian) 
[13] M. Kimura, S. Dost, H. Udono, A. Tanaka, T. Sukegawa and Z. Qin. A numerical analysis for the conversion phenomenon of GaAs to GaAsP on a GaP substrate in an LPE system. Journal of Crystal Growth, 169(1), 697 - 703, 1996.

[14] V.V. Kolmychkov, O.S. Mazhorova and Yu.P. Popov. On the numerical solution of Navier-Stokes equations in primitive variables. The Keldysh Institute of Applied Mathematics, Preprint 60,Moscow, 2001. (in Russian)

[15] V.V. Kolmychkov, O.S. Mazhorova and Yu.P. Popov. Mathematical modelling for convective mass transfer in $3 D$ case, part 1 . The Keldysh Institute of Applied Mathematics, Preprint 92, Moscow, 2003. (in Russian)

[16] V.V. Kolmychkov, O.S. Mazhorova and Yu.P. Popov. Mathematical modelling for convective mass transfer in $3 D$ case, part 2. The Keldysh Institute of Applied Mathematics, Preprint 98, Moscow, 2003. (in Russian)

[17] R. Krishnamurti. Finite amplitude convection with changing mean temperature. Part I. Theory. Journal of Fluid Mechanics, 33, 445 - 455, 1968.

[18] R. Krishnamurti. Finite amplitude convection with changing mean temperature. Part II An experimental test of the theory. Journal of Fluid Mechanics, 33, $457-463,1968$.

[19] R. Krishnamurti. Further studies on transition to turbulent convection. Journal of Fluid Mechanics, 60, 285 - 304, 1974.

[20] W.V. Malkus and G. Veronis. Finite amplitude cellular convection. Journal of Fluid Mechanics, 4, 225 - 260, 1958.

[21] O.S. Mazhorova, Yu.P. Popov and A.S. Sakharchuk. Stability of finite difference schemes for the systems of parabolic equations with non - traditional boundary conditions. Differential equations, 33(7), 946 - 954, 1997. (trans. from Russian)

[22] G. Müller. Crystal growth from the melt. Convection and inhomogeneities. Mir, Moscow, 1991. (in Russian)

[23] A. Schlüter, D. Lortz and F. Busse. On the stability of steady finite amplitude convection. Journal of Fluid Mechanics, 23, 129 - 144, 1965.

[24] K. Stork and U. Müller. Convection in boxes: experiments. Journal of Fluid Mechanics, 54, 599 - 611, 1972.

[25] V.B. Ufimtsev and R.H. Akchurin. Physical and chemical principals of liquid phase epitaxy. Metallurgiya, Moscow, 1983. (in Russian)

[26] P.K. Vinsome. An ORTHOMIN, an iterative method for solving sparse set of simultaneous linear equations. In: Proc. 4 Symp. Reservoir simulation. Soc. Petroleum Eng. AIME, 149 - 159, 1976.

[27] H.R. Vydyanath. Status of $\mathrm{Te}$ - rich and $\mathrm{Hg}$ - rich liquid phase epitaxial technologies for growth of $(\mathrm{Hg}, \mathrm{Cd}) \mathrm{Te}$ alloys. Journal of Electronic Materials, 24(9), 1275 - 1285, 1995.

[28] D.B. White. The planforms and onset of convection with a temperaturedependent viscosity. Journal of Fluid Mechanics, 191, 247 - 286, 1988.

[29] S. Zaleski, Y. Pomeau and A. Pumir. Optimal merging of rolls near a plane boundary. Physical Review A, 29(1), 366 - 370, 1984. 